Case report

\title{
Tongue reconstruction using VRAM flap after staged neck dissection and total glossectomy for squamous cell carcinoma
}

\author{
Liviu Dumitru Damian ${ }^{*, 1}$, Atena Florina Aanicăi ${ }^{1}$, Alina Chelmuș ${ }^{1,2}$, Alexandru Mihai ${ }^{1}$, \\ Dan Cristian Moraru ${ }^{1,2}$, Codrin Nicolae Dobreanu ${ }^{1}$
}

${ }^{1}$ Plastic and Reconstructive Surgery Department, Regional Institute of Oncology, Iași, Romania; ${ }^{2}$ Department of Surgery, "Grigore T. Popa" University of Medicine and Pharmacy, Iași, Romania

\begin{abstract}
Squamous cell carcinoma (SCC) is a frequent cancer of the oral cavity, more aggressive in young patients and mostly affecting males with a history of smoking and alcohol consumption. After oncological excision, reconstruction is possible using an array of flaps, such as the radial forearm flap, anterolateral thigh flap, rectus abdominis flap, each with various indications, advantages and disadvantages. Functional reconstruction is difficult, as the multiple and highly specialized muscles of the tongue cannot be replaced by any of the abovementioned options. A 23 year-old male presented with squamous cell carcinoma of the anterior $2 / 3$ of the tongue and left submandibular enlarged lymph nodes $\left(\mathrm{T}_{3} \mathrm{~N}_{2 b} \mathrm{M}_{0}\right.$, stage IVA). A preoperative nutritional and metabolic optimization was established using a percutaneous endoscopic gastrostomy, tracheostomy, then we performed a total glossectomy with modified radical bilateral neck dissection and VRAM flap reconstruction. Postoperatively the patient developed an orocervical salivary fistula and partial skin necrosis of the upper left cutaneous laterocervical flap. The postoperative pathology confirmed extended SCC of the tongue. The patient received adjuvant radiotherapy postoperatively. There were no major complications, and the one-year followup concluded on normal feeding, satisfactory speech recovery, and no recurrence.
\end{abstract}

Keywords: tongue squamous cell carcinoma; neck dissection; tongue reconstruction; VRAM; free flap

\section{Introduction}

Squamous cell carcinoma (SCC) is one of the most frequent oral cancers, but even though it is uncommon in patients younger than 40 years' old, occurring mostly in elderly man, a slight increase in the number of cases in the young group was noted. A review of the literature indicates that the SCC of the tongue has a prevalence of $20 \%$ of all the carcinomas

Received: July 2018; Accepted after review: September 2018; Published: September 2018.

${ }^{*}$ Corresponding author: Liviu Dimitrie Damian MD, Department of Surgery, Regional Oncology Institute, lasi, Romania.

Email: damian.liviu.d@gmail.com of the oral cavity, $75 \%$ affecting the anterior $2 / 3$ and lateral borders of the tongue [1-3].

Considering both functional and esthetic importance of the head and neck, the reconstruction of these regions proves to be challenging. The tongue is a key organ, being involved in nutrition and speech, and, as such, it requires a thorough approach in restoring its function after glossectomy. Total glossectomy is defined by the complete resection of the tongue, sub-total glossectomy by the removal of more than $2 / 3$, and hemi-glossectomy the resection of half of the tongue or less $[1,3,4]$.

When deciding upon an oncologic excision technique, the surgeon must take into consideration the location of the tumor. As for reconstruction, the possibilities are: primary closure (if the defect is less than $50 \%$ of the 
tongue) or possible donor sites for the free flaps, depending on the patient's health status, recovery time and desired results [5].

For large glossectomy defects the surgeon is aiming to close the defect, minimize complications, and optimize speech and deglutition. Local or regional flaps are insufficient for properly restoring the tongue and its functions after total glossectomy, and thus the need for free tissue transfer. Providing both enough bulk and pliability, free flaps are the workhorse for tongue reconstruction $[1,5,6]$.

\section{Case report}

A 23 year-old male, with 4 packs/year history of smoking, was referred to our department for left submandibular swellings and impaired swallowing and phonation due to a 4-year history of a progressive growing tumor of the tongue, involving the anterior $2 / 3$ of the tongue. The patient's BMI was $16.7 \mathrm{~kg} / \mathrm{m}^{2}$, as a result of hindered feeding. Local examination revealed a $4.5 \times 4 \times 3.5 \mathrm{~cm}$ ulceroproliferative tumor (Figure 1) that split the tongue in the midline (Figure 2).

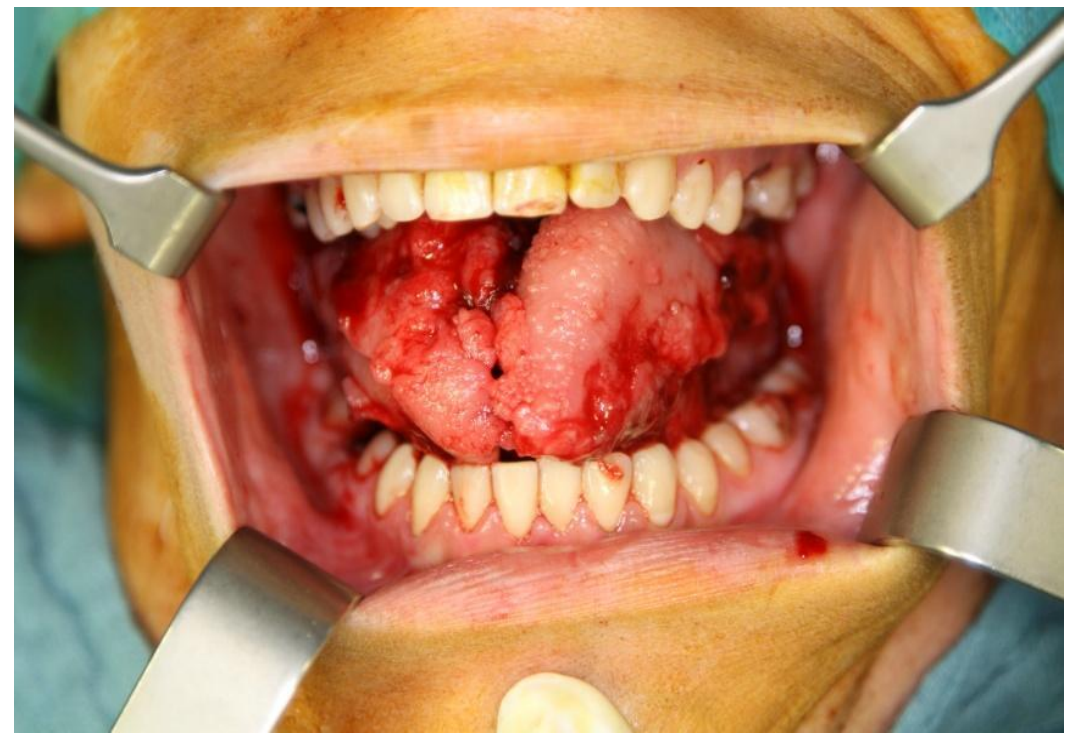

Fig. 1. The ulcerated tumor of the tongue.

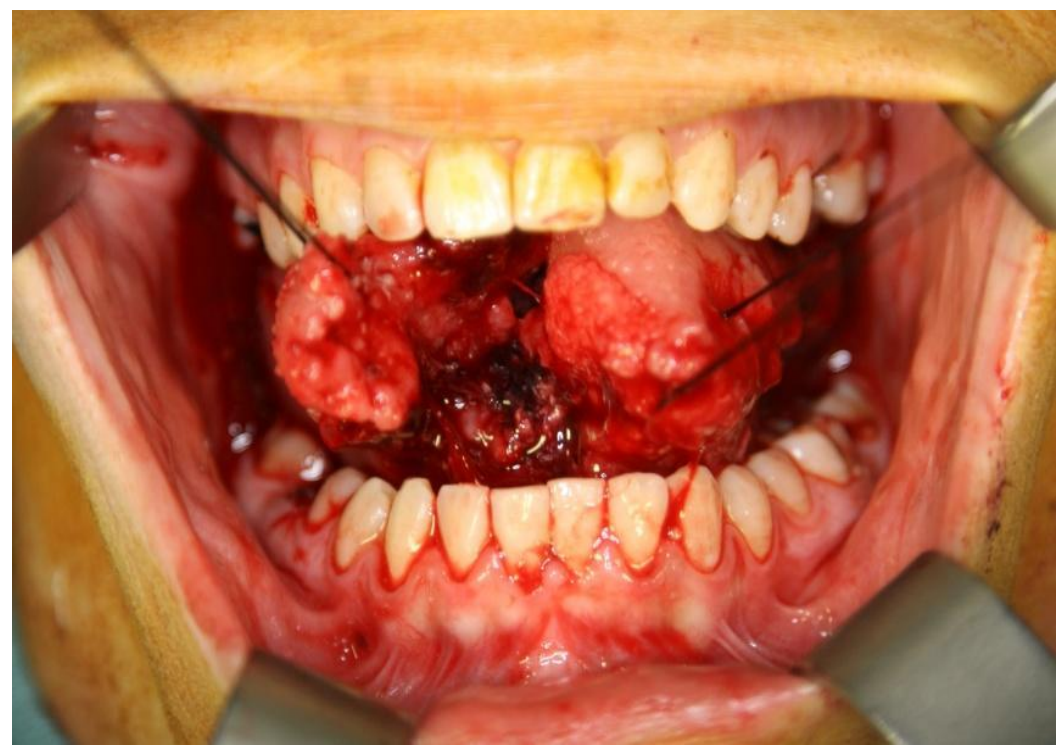

Fig. 2. The ulcero-prolifrative tumor split the median septum of the tongue. 
An incisional biopsy of the tumor confirmed the diagnosis of squamous cell carcinoma. The results of a following CT scan identified a 4.5/4/3.5 $\mathrm{cm}$ tumor involving the anterior $2 / 3$ of the tongue and left latero-cervical enlarged lymph nodes. According to NCCN staging guidelines, the tumor was staged as $\mathrm{T}_{3} \mathrm{~N}_{2 b} \mathrm{M}_{0}$ (IVA in the TNM 2014 staging) [7].

The oncologic committee decided on preoperative nutritional optimization to restore a normal BMI, followed by oncologic excision, with bilateral neck dissection and appropriate reconstruction. Due to low $\mathrm{BMI}$ and altered metabolic values, a percutaneous endoscopic gastrostomy (PEG) tube was inserted (February 2014) and the patient was placed on an intense feeding regimen, prior to surgery. In March 2014, the patient was admitted to the Plastic Surgery Department, but he was dismissed due to a preoperative panic attack, under strict surveillance of a psychologist.

Two weeks later, in April 2014, the patient was admitted for a 2-stage treatment plan: neck dissection and tumor removal with tongue reconstruction. A tracheostomy below the thyroid isthmus and bilateral neck dissection, preserving the spinal accessory nerve, internal jugular vein and sternocleidomastoid muscle, were performed (Figures 3 and 4).

The intraoperative histopathological examination of the lymph nodes was positive for tumor invasion in lymph nodes groups I-IV bilaterally.

Two days later, excision of the anterior $3 / 4$ of the tongue along with the neoplastic mass through a transmandibular paramedian approach, resection of the mouth floor and sublingual glands (Figures 5 and 6 ).

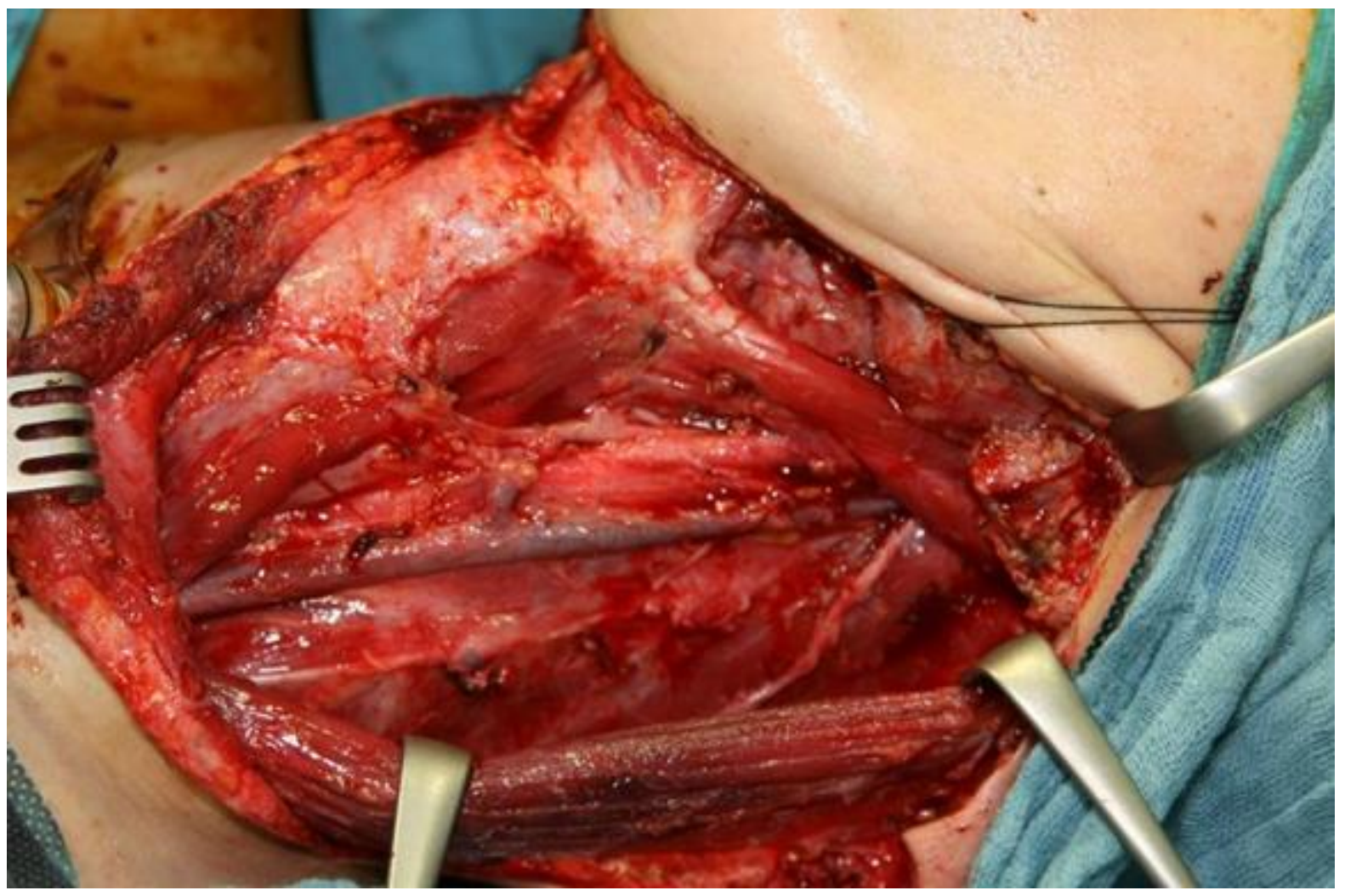

Fig. 3. The anterolateral left cervical region after neck dissection with preservation of the spinal accessory nerve, internal jugular vein and sternocleidomastoid muscle. 


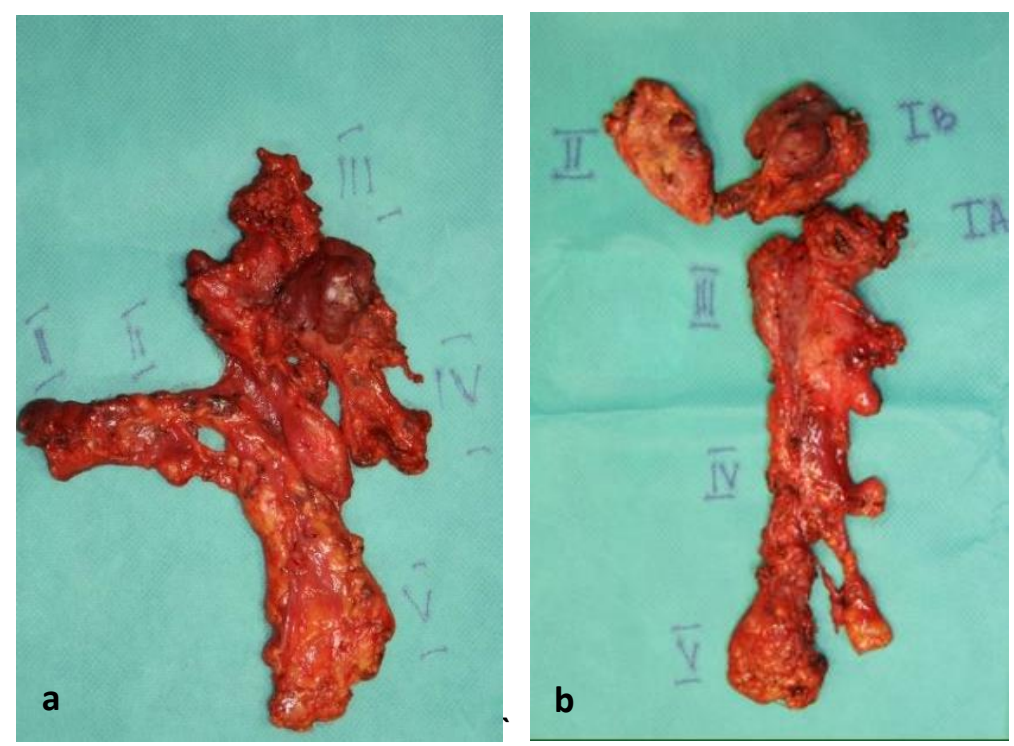

Fig. 4. Specimens of bilateral neck dissection with preservation of the spinal accessory nerve, internal jugular vein and sternocleidomastoid muscle. (a) Right cervical lymph nodes. (b) Left cervical lymph nodes.

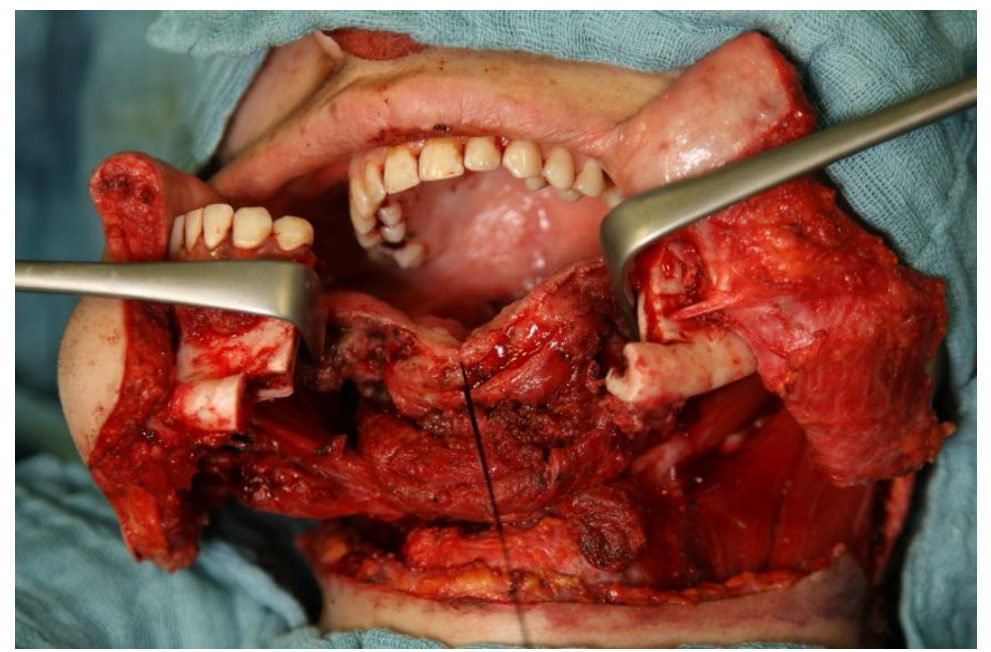

Fig. 5. The resulting defect after the tongue carcinoma excision, about $7.5 / 5 / 5 \mathrm{~cm}$.

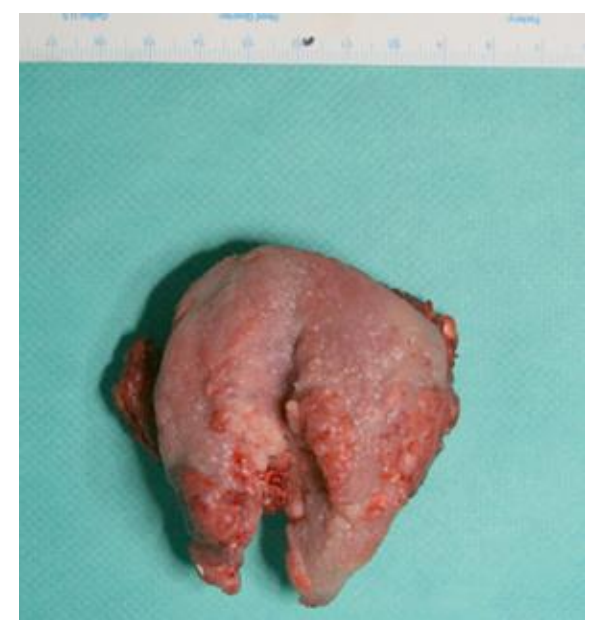

Fig. 6. Superior view of the excised tongue specimen. At the inferior side of the specimen the split median septum can be identified. 
Immediate reconstruction of the tongue using a free flap was tempted, in order to restore speech and swallowing as quickly as quickly as possible. The VRAM flap, based on the left deep inferior epigastric pedicle, was designed with a skin island of $11 \times 10 \mathrm{~cm}$ and a muscular component of $16 \times 9 \mathrm{~cm}$ (Figure 7). Microvascular end-to-end anastomosis was performed in the left cervical region, to the superior thyroid artery, respectively to a branch of the internal jugular vein, under microscope magnification (Figure 8).

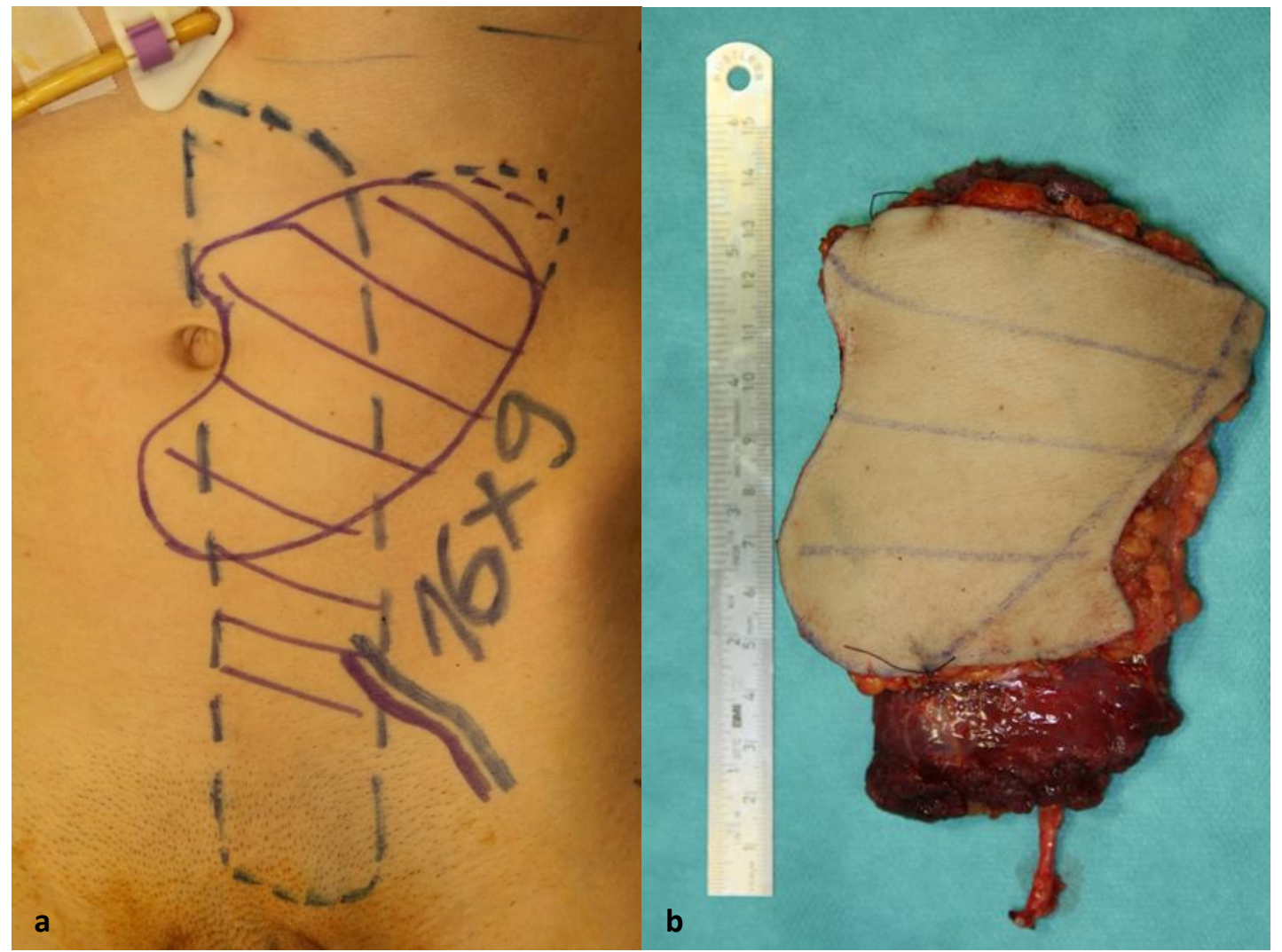

Fig. 7. (a)The flap design on the left side of the abdomen includes a skin island of $16 / 9 \mathrm{~cm}$, a muscle size of $16 / 9$ $\mathrm{cm}$; (b) the elevated free flap on the left deep inferior epigastric pedicle.

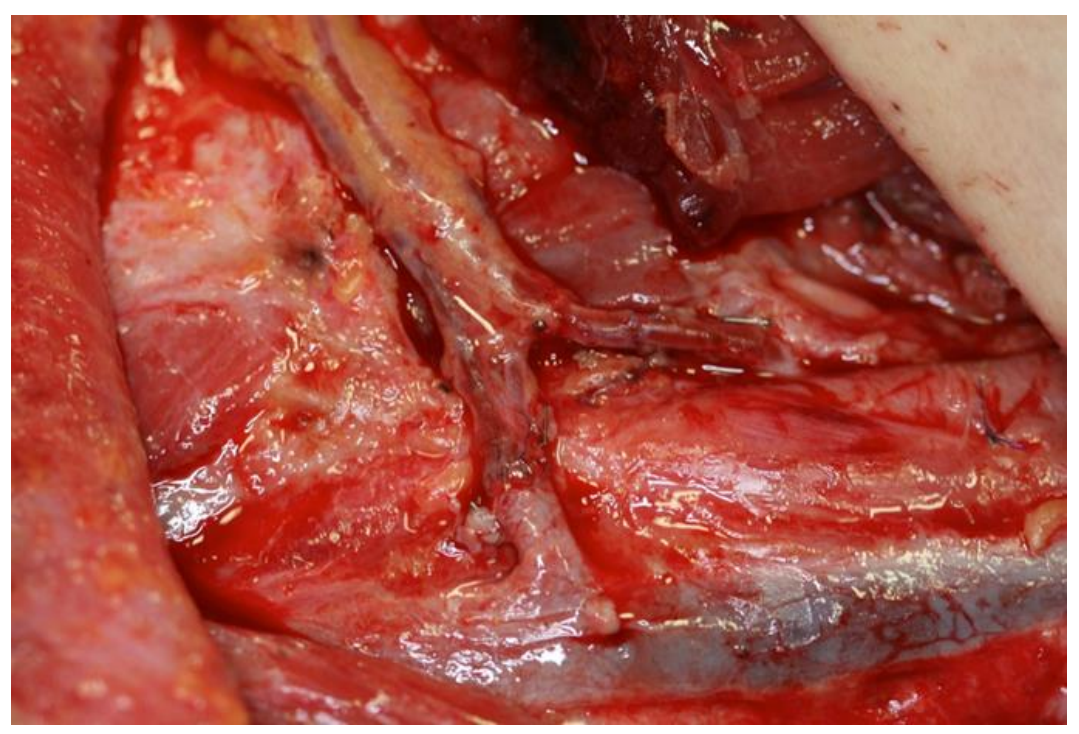

Fig. 8. Microvascular anastomosis in the left cervical region, end-to-end between the left deep inferior epigastric pedicle and the superior thyroid artery, and between the pedicle vein and a branch of the internal jugular vein, respectively. 
The muscle flap was inset in the midline to create the neo tongue projection, while the three-lobed skin paddle and the lateral aspects of the flap were used to reconstruct the lingual sulcus, using circumdental sutures due to the absence of the mucosa. The final shape of the reconstructed tongue was bulging, having a size overcorrection of $30 \%$ (Figure 9). Mandibular osteosynthesis was performed using a plate and screws.

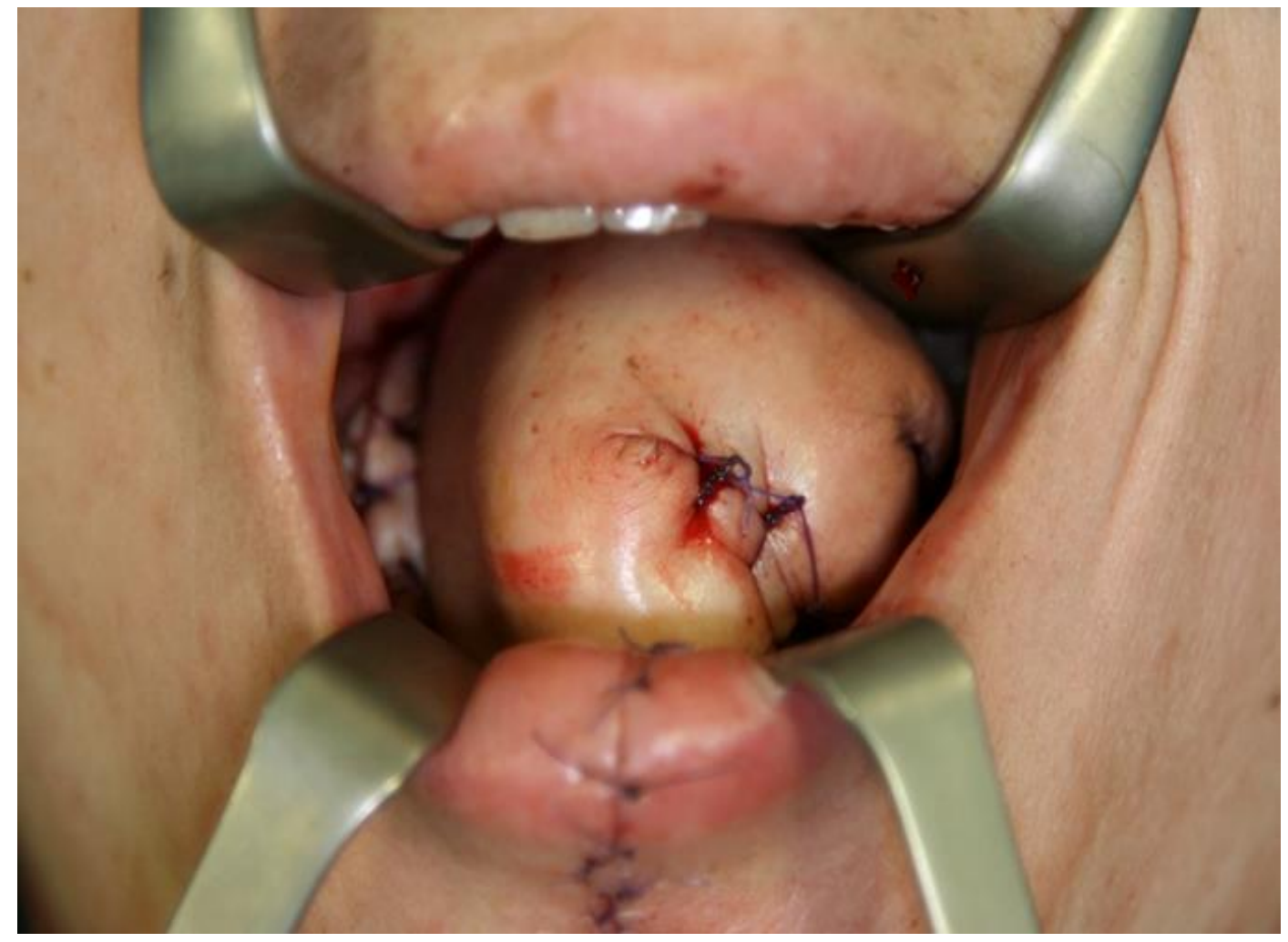

Fig. 9.The final outcome of the flap design, forming a bulging shape of the new tongue.

The resulting abdominal defect was closed using a component separation technique and a right anterior fascial rectus abdominis flap. The umbilicus was dissected and separated from the surrounding flap and soft tissue and reinserted on the medial line.

The excised neoplastic mass $(7.5 \times 5 \times 5 \mathrm{~cm})$ was sent for intraoperative histopathological examination, and the result was that of squamous cell carcinoma with free circumferential and deep excision margins.

Immediate postoperative complications were orocervical salivary fistula, developed in the 4th postop day, and skin necrosis on the upper part of the left laterocervical flap, with consequential wound dehiscence on the $7^{\text {th }}$ day, due to patient noncompliance who smoked throughout the hospital stay. The fistula was closed using a vestibular mucosal flap in the $11^{\text {th }}$ postoperative day, due to the high-risk of flap necrosis and infection at the site of the mandibular osteosynthesis. During the same procedure, the neck skin defect was reduced in size.

The patient was dismissed in the 12th day post-surgery, with a fully integrated free flap and granular laterocervical wounds (Figure 10).

The final histopathological resulted in one positive lymph node for both $3^{\text {rd }}$ and $4^{\text {th }}$ left lymph node groups. The histological examinations of the tumor confirmed tongue squamous cell carcinoma, moderate differentiation, keratinization, with large areas of ulcers, intense pleomorphism, many mitosis, perineural infiltration, $\mathrm{pT}_{3} \mathrm{~N}_{\mathrm{x}} \mathrm{G} 2$. The tumor was excised with negative resection margins, the closest margin being at $0.4 \mathrm{~cm}$ measured post formalin fixation. 


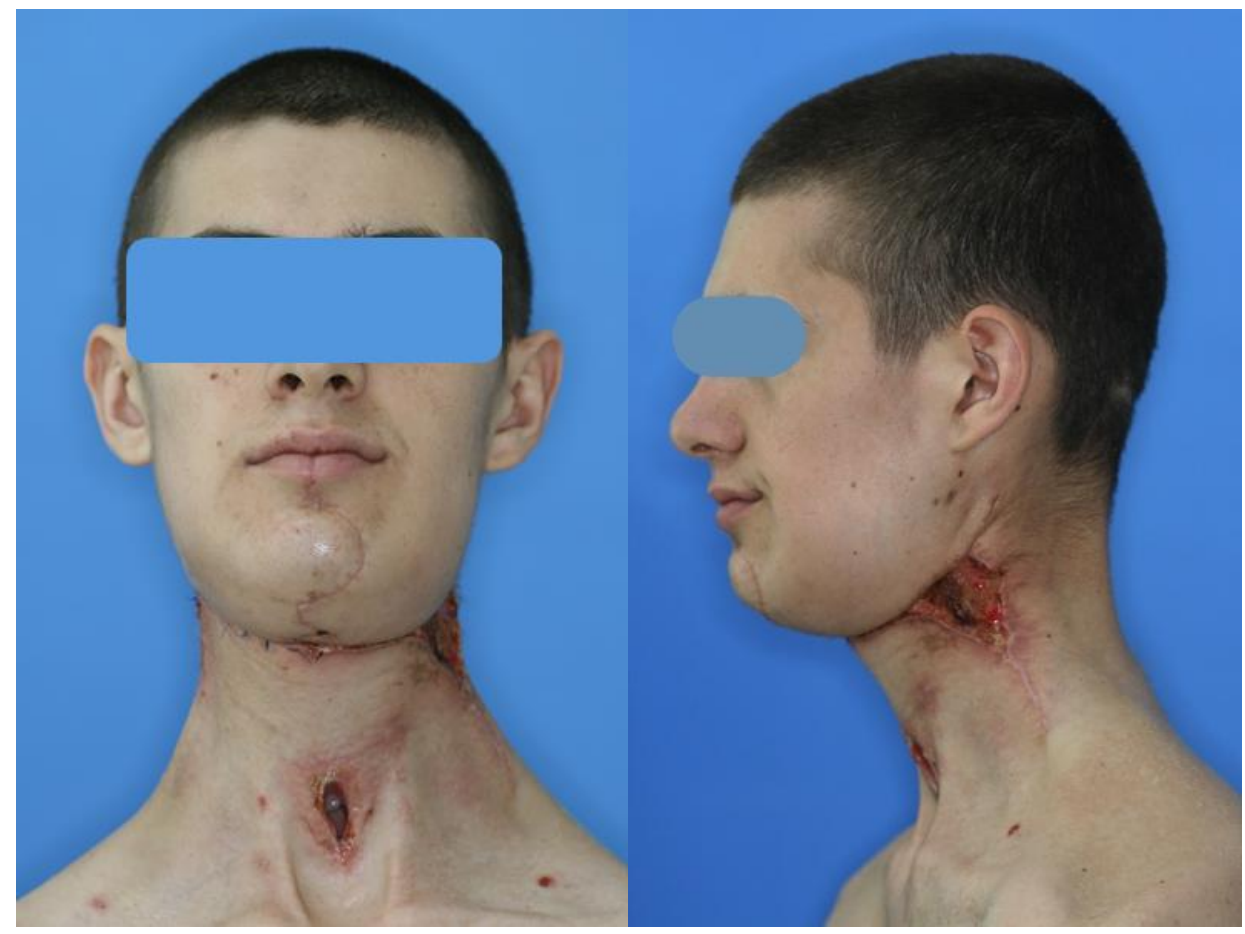

Fig. 10. The outcome at 1 month, with partial skin necrosis closed with secondary healing due to the noncompliance of the patient.

In May 2014, the patient was admitted for tracheostomy closure. The PEG tube remained in place for an additional four weeks, until normal feeding process could be resumed. During the follow up, swallowing was evaluated using videofluoroscopy and revealed a normal oropharyngeal passage, without any fistulas or additional opaque residue. The local examination of the reconstructed tongue identified a near normal neotongue shape with a higher projected tip and vertical bulk allowing it to come into contact with the palate and the upper lip, thus facilitating swallowing (Figures 11 and 12).

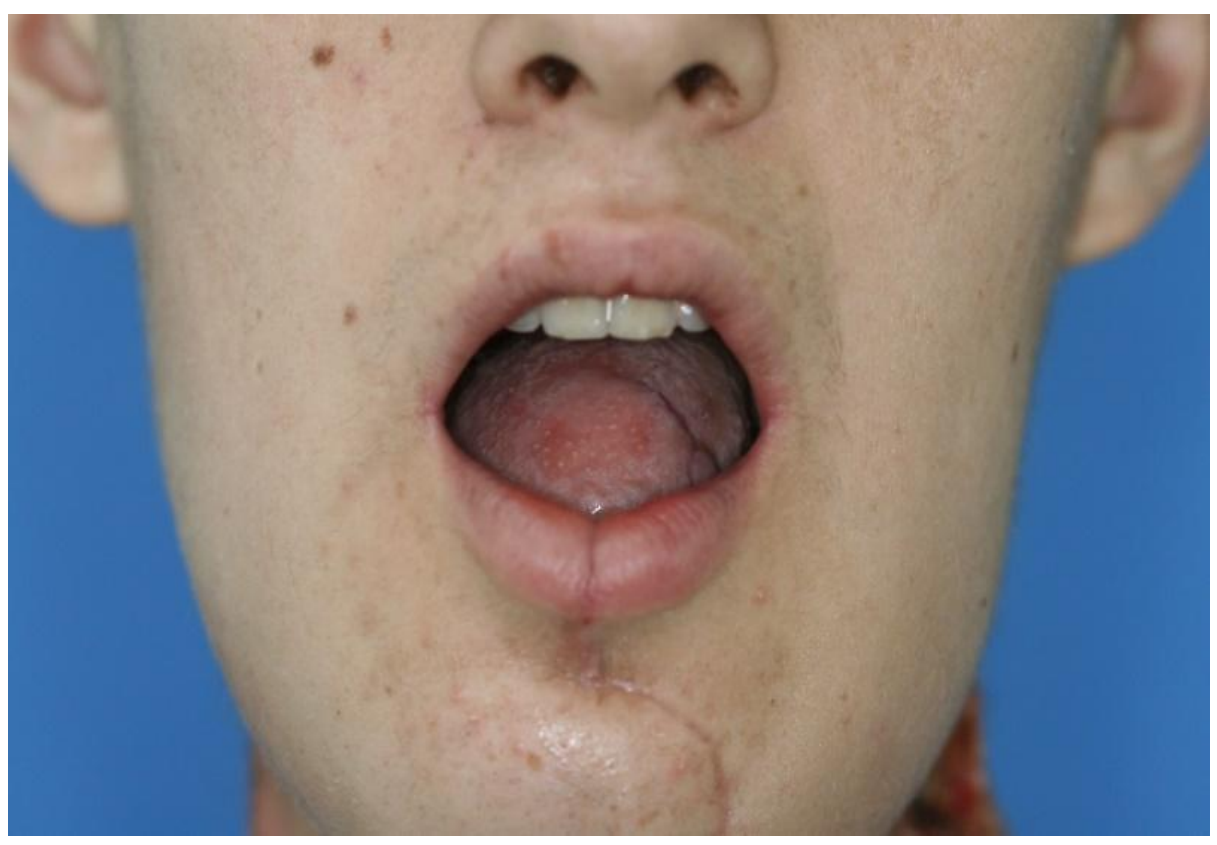

Fig.11. Mucolization of skin paddle of the flap. The outcome at 1 month, with the neotongue shape with projected tip and vertical bulk. 


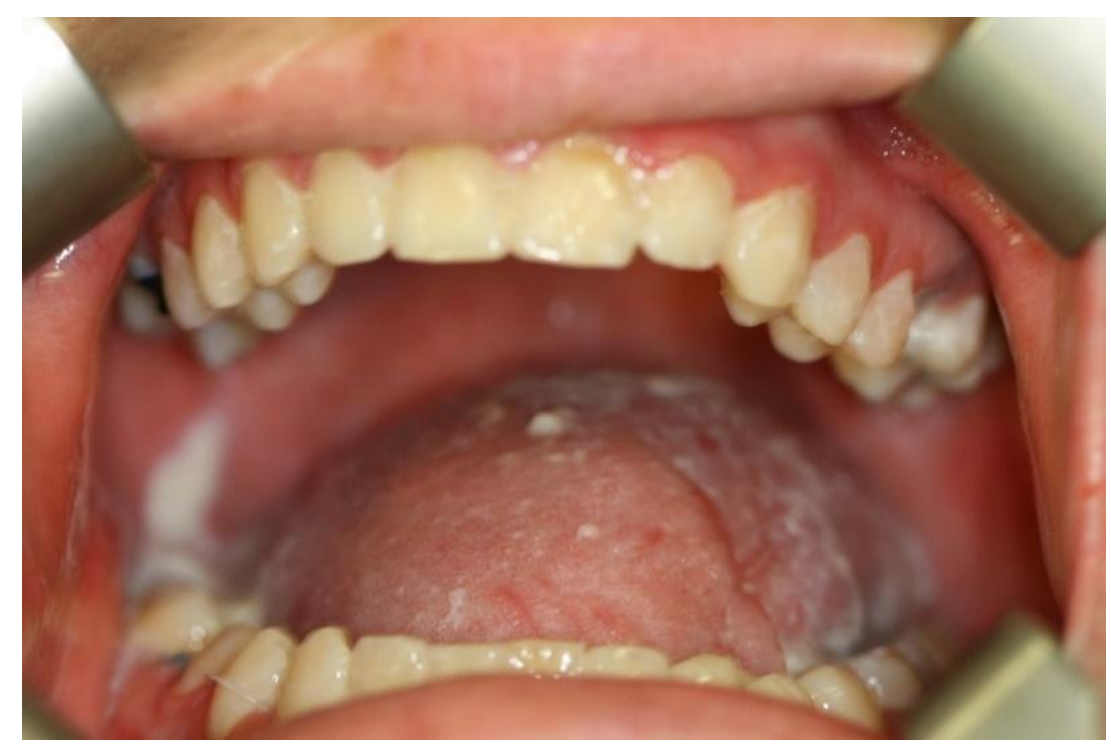

Fig. 12. The outcome at 1 month, neotongue shape with projected tip and vertical bulk.

Because of the $T_{3}$ tumor staging, $\mathrm{N}_{2}$ nodal disease, bilateral level IV nodal disease and perineural infiltration, postoperative radiotherapy was necessary. The patient was irradiated with $60 \mathrm{~Gy} / 30 \mathrm{fr} / 6$ weeks for the tongue and $50 \mathrm{~Gy} / 25 \mathrm{fr} / 5$ weeks for the neck region. Fractionation allows normal cells time to recover, while tumor cells are generally less efficient in repair between fractions. At the time of radiotherapy the patient had normal $\mathrm{BMI}$, but continued smoking, ignoring our recommendations. Early complications after radiotherapy included small sized ulcerations, partial neck skin flap necrosis and no flap loss. Later complications included fibrosis and volume loss. The irradiated neotongue had a small volume loss, but still able to touch the palate and have a satisfying swallowing function.

The patient was lost for follow up after one year, and three years later it was discovered that the patient unfortunately had passed away due to malignancy.

\section{Discussions}

In past years, the squamous cell carcinoma of the tongue was considered to be a disease encountered mostly in elderly patients, but in recent years a large number of cases in patients under 40 years old have been reported. Although, alcohol and tobacco consumption are considered risk factors in older patients, the most recent studies did not reveal any statistically significant correlations between younger ages and alcohol and tobacco consumption [2, 8, 9].

The development of the pectoralis major myocutaneous flap was a breakthrough in tongue reconstruction, and, together with the development of free flaps such as gracillis, radial forearm, anterolateral thigh and RAM flap (rectus abdominis muscle flap) optimized subtotal and total tongue reconstruction [4,1012].

The extent of the SCC required a twoteam approach, one for resecting the tumor and another for raising the flap. In our patient, the first three flaps do not provide enough bulk for a total glossectomy reconstruction and as for the LD flap, the patient needs to be positioned in lateral decubitus, leaving the VRAM flap as the best reconstruction option $[5,10,12]$.

The VRAM flap is favored in head and neck reconstruction since it is easy to harvest, has a long vascular pedicle and anatomic consistency, and provides well vascularized tissue that can survive irradiation. This flap provides enough tissue bulk for a shaped neotongue, since an overcorrection of at least $40 \%$ is needed if postoperative radiotherapy is involved. It has also been demonstrated that patients with normal shaped neotongues have excellent functional recovery [3]. The VRAM 
flap allows a wide variety of skin flap designs that can be tailored to fit most defects reconstruction of the exterior side of the tongue and the sublingual folds; if the cutaneous island flap is raised more cephalad it provides a longer pedicle and it is easier to reshape $[1,3,5,6,13]$.

Tongue innervation was not a priority in this case since the patient was ineligible, the VRAM flap provided enough overcorrection, and, also, the postoperative irradiation and the prognostic of the patient had to be taken into account.

Study results vary when it comes to complex tongue reconstruction, some reporting good uniform results, whereas others report patients having poor speech intelligibility, swallowing dysfunction or problematic drooling $[1,5,9,14,15]$. Stage IV oral tongue cancer patients show a 5-year survival rate of $30-65 \%$ in patients that had undergone surgical treatment and a 11-40\% 5year survival rate in patients with nonsurgical treatment $[4,16]$.

\section{Conclusion}

Patients with squamous cell carcinoma of the tongue necessitate a multidisciplinary treatment approach in order to get satisfactory

\section{References}

1. Leymarie N, Karsenti G, Sarfati B, Rimareix F, Kolb F. Modification of flap design for total mobile tongue reconstruction using a sensitive antero-lateral thigh flap. J Plastic Reconstruct Aesthetic Surg 2012; 65:e169ee174.

2. Friedlander PL, Schantz SP, Shaha AR, Yu G, Shah JP. Squamous cell carcinoma of the tongue in young patients: a matched-pair. Head Neck 1998; 20:363-368.

3. Sakuraba M, Asano T, Shimpei M, Hayashi R, Yamazaki M, Miyazaki M, Ugumori T, Daiko H, Kimata Y. A new flap design for tongue reconstruction after total or subtotal glossectomy in thin patients. $J$ Plastic Reconstruct Aesthetic Surg 2009; 62, 795e799.

4. Vega C, Leon X, Cervelli D, Pons G, Lopez S, Fernandez M, Quer M, et al. Total or subtotal glossectomy with microsurgical reconstruction: esthetic and functional results, as well as good survival rates. The young age of the patients does not exclude malignancy, and tissue biopsy is needed to provide an accurate diagnosis. Reconstruction of the tongue using the VRAM flap proves to be a reliable solution, providing the muscle bulk necessary for swallowing and speech recovery and enough skin for recreating the surface of the tongue and sulcus. Common postoperative complications are orocutaneous fistulas and partial skin necrosis of the laterocervical advancement flaps. Alcohol and tobacco consumption are important risk factors for oral cancer development and thus the need to prevent abuse in the young population group [17]. Survival rates at 2 years vary in young patients (under 40 years) from $45 \%$ to $78 \%$, under strict follow-up and proper treatment. These rates are similar to those found in patients older than 40 years [8].

\section{Consent}

Written informed consent was obtained from the patient for publication of this case report.

\section{Competing interests}

The authors declare that they have no competing interests.

functional and oncological results. Microsurgery 2011; 31(7):517-523.

5. Lyos AT, Evans GRD, Perez D, Schusterman MA. Tongue reconstruction: outcomes with the rectus abdominis flap. Plastic Reconstr Surg 1999; 103(2):442-447.

6. Lam L, Samman N. Speech and swallowing following tongue cancer surgery and free flap reconstruction - a systematic review. Oral Oncol 2013; 49:507-524.

7. National Comprehensive Cancer Network (NCCN) Clinical Practice Guide in Oncology, Head Neck Cancers, version 2, 2017. [https://www.nccn.org/professionals/physician_ gls/default.aspx available at 10/17/2018]

8. Myers JN, Elkins $\mathrm{T}$, Roberts $\mathrm{D}$, Byers RM. Squamous cell carcinoma of the tongue in young adults: increasing incidence and factors 
that predict treatment outcomes. Otolaryngol Head Neck Surg 2000; 122:44-51.

9. Zygogianni AG, Kyrgias G, Karakitsos P, Psyrri A, Kouvaris J, Kelekis N, Kouloulias V. Oral squamous cell cancer: early detection and the role of alcohol and smoking. Head Neck Oncol $2011 ; 3: 2$.

10. Vural E. Surgical reconstruction in patients with cancer of the head and neck. Current Oncol Rep 2004; 6:133-140.

11. Wong $\mathrm{CH}$, Wei FC. Microsurgical free flap in head and neck reconstruction. Head Neck 2010; 32(9):1236-1245.

12. Engel $\mathrm{H}$, Huang JJ, Lin $\mathrm{CY}$, et al. A strategic approach for tongue reconstruction to achieve predictable and improved functional and aesthetic outcomes. Plast Reconstr Surg 2010; 126:1967-1977.

13. Matsui $Y$, Shirota $T$, Yamashita $Y$, Ohno $K$. Analyses of speech intelligibility in patients after glossectomy and reconstruction with fasciocutaneous/myocutaneous flaps. Int $J$ Oral Maxillofac Surg 2009; 38:339-345.

14. Pirgousis $P$, Fernandes R. Current therapy in oral and maxillofacial surgery. In Bagheri SC, Bell RB and Khan HA (Ed). Contemporary methods in tongue reconstruction. Philadelphia: Elsevier Saunders, 2012.

15. Dassonville O, Poissonnet G, Chamorey E, et al. Head and neck reconstruction with free flaps: a report on 213 cases; Eur Arch Otorhinolaryngol 2008; 265:85-95.

16. Sessions DG, Spector GJ, Lenox J, Haughey B, Chao C, Marks J. Analysis of treatment results for oral tongue cancer. Laryngoscope 2002; 112(4):616-625.

17. Hart AK, Karakla DW, Pitman KT, Adams JF. Oral and oropharyngeal squamous cell carcinoma in young adults: a report on 13 cases and review of the literature. Otolaryngol Head Neck Surg 1999; 120(6):828-833. 